\title{
ACOUSTIC PLANE WAVE REFLECTION FROM A COMPOSITE LAMINATE: NORMAL INCIDENCE
}

\author{
A. BAGCHI \\ Department of Applied Mathematics and Mechatronics Research Centre Twente, \\ University of Twente, P.O. Box 217, 7500 AE Enschede, The Netherlands
}

AND

S. K. Bose $\dagger$

Department of Mathematics, Regional Engineering College, Durgapur, West Bengal, India

(Received 24 July 1992, and in final form 7 December 1992)

\begin{abstract}
In using ultrasonic non-destructive evaluation methods for composite laminates, some features appear which are essentially due to the heterogeneity of the material. In performing backscattering phenomena, one such feature is the seemingly random backscattered amplitude with change of position in a $c$-scan. This is attributed to the local heterogeneity due to slight changes in position, orientation and bonding characteristics of the fibers. In the case of incidence normal to the laminate, the average reflection coefficient, containing the backscattered waves from the fibers, is determined in terms of the fiber concentration and the properties of the fiber and matrix phases. The case of thin fibers, such as those of glass and carbon, in a polymeric matrix is also an assumption in the theory. Next, attention is paid to the actual random backscattered reflection coefficient, to estimate it at any location from experimental data at a given grid of locations of the lamina. The method used is called "kriging" in mining geostatistics.
\end{abstract}

\section{INTRODUCTION}

Ultrasonic non-destructive evaluation methods, originally developed to detect single flaws in homogeneous and isotropic, monolithic materials are increasingly being recommended for evaluating composite laminates. On the one hand, the "leaky Lamb wave" method and, on the other, the "backscattering imaging" method, are being developed for detecting subcritical flaws in such laminates. In experiments in which these methods are used, the laminate is immersed in a water bath (the water acting as a couplant and a delay line) and either (i) a tone-burst waveform at a selected frequency is sent or (ii) a pulse is generated in a given frequency $(\mathrm{MHz})$ range $[1,2]$.

In the backscattering imaging method, the second method has been used, where some special features have been observed in the received signal [3]. One of these relates to practically random amplitudes in a $c$-scan. The reason may be seen in the fact that the backscattered beam carries information about the properties of the location from where it is emergent. A possible slight change in the position of the fibers, their alignment and the extent of bonding with the matrix can significantly change the backscattered pulse.

As a prelude to using the above phenomenon for flaw detection in a composite laminate we suppose that the prober is normal to the laminate, which has unidirectional fibers within

† This work was performed when the author was visiting the Department of Applied Mathematics and Mechatronics Research Centre Twente, University of Twente, on leave from the Regional Engineering College, Durgapur, India. 
it. We assume that the probe generates a wavefront, which is nearly a plane, while striking the spot of the laminate being probed and has a certain high frequency content $\omega / 2 \pi$. We shall thus have the backscattered wave as a part of the total reflected wave, which we call the backscattered reflected wave. The amplitude of the wave, being location dependent, is random and we seek the average reflection coefficient. The method adopted is that of Bose and Mal [4] for determining the average properties of compressional and shear waves propagating across a fiber reinforced composite. It may be noted that, although we have considered a unidirectional composite, the calculated average backscattered amplitude will not differ when the laminate has several plies with fiber in different orientations. This assertion stems from the assumed direction of wave propagation, normal to the laminate and thus also normal to the fibers.

A second question that we have studied is the estimation of the backscattered reflection coefficient at any location, when experimental values at a grid of locations are known. This question is imperative, because a database of the coefficient can be prepared in this way for an undamaged laminate, which may be used for comparison when the laminate develops a flaw at some location. This statistical problem arose, and is widely used, in mining geostatistics [5]. The procedure is explained in our present context.

\section{SYNTHESIS OF BACKWARD REFLECTION AND SCATTERING AMPLITUDES}

We designate the physical quantities associated with water by subscript 0 and those with fibers by primes. Those without these symbols are for the matrix material. We assume that a single frequency plane wave, travelling in water, is incident normally to the left face of the composite laminate (see Figure 1). With the laminate considered as a slab of matrix material containing the scattering fibers, a part of the energy is transmitted and a part is reflected. If the transmission and reflection coefficients are $T$ and $R_{0}$, then since no shear wave is generated because of normal incidence, it is easy to show that

$$
T=2 \rho_{0} \alpha /\left(\rho \alpha+\rho_{0} \alpha_{0}\right), \quad R_{0}=\left(\rho \alpha-\rho_{0} \alpha_{0}\right) /\left(\rho \alpha+\rho_{0} \alpha_{0}\right),
$$

where $\alpha, \rho$ and $\alpha_{0}, \rho_{0}$ are the velocity and density in the matrix and the water, respectively, $\left(\alpha=(\lambda / \rho)^{1 / 2}, \alpha_{0}=\left(\lambda_{0} / \rho_{0}\right)^{1 / 2}, \lambda, \mu\right.$ being Lamé constants of the matrix material, and similarly for $\lambda_{0}$ of water). The transmitted wave encountering the scattering fibers is partially backscattered and partially forward-scattered, as will be shown in the next section. The

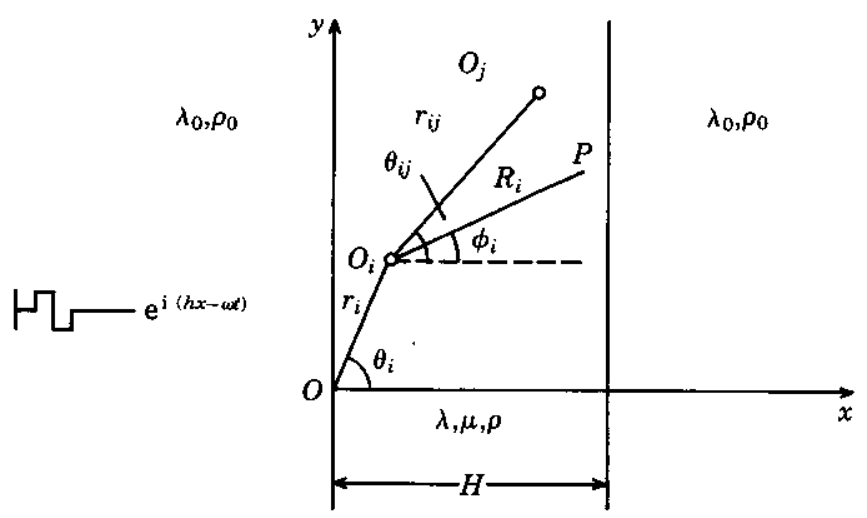

Figure 1. Schematic of a plane wave incident normally to a composite laminate containing unidirectional fibers, immersed in water. 


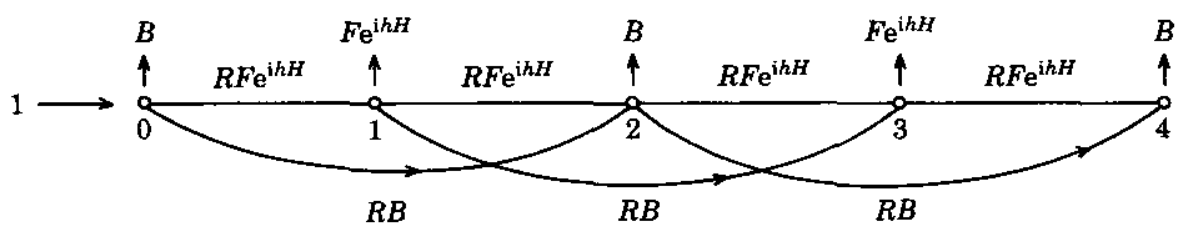

Figure 2. Signal flow graph of successive reflections.

forward-scattered wave is partially reflected by the right interface with the water, where the reflection and transmission coefficients are now given by

$$
R=-R_{0}, \quad T_{0}=2 \rho \alpha_{0} /\left(\rho \alpha+\rho_{0} \alpha_{0}\right) .
$$

Let us suppose that the backscattering and the forward-scattering coefficients are $B$ and $F$, respectively. Expressions for these are derived in section 4 (equations (43) and (45)), in terms of the matrix and fiber properties and their concentration $c$ - the fractional area occupied by the fibers in a cross-section. The multiply reflected wave between the two faces of the slab can be represented by a signal flow graph. If we denote the first incidence and the succeeding reflections by the nodes $0,1,2,3, \ldots$ with transmission coefficient $T$ associated with node 0 and reflection coefficients $R_{1}, R_{2}, R_{3}, \ldots$ with the rest of the nodes, then the graph is as shown in Figure 2. Thus, the reflection coefficients are given by

$$
\begin{array}{r}
R_{1}=R F \mathrm{e}^{\mathrm{i} h H} T, \quad R_{2}=R\left(F \mathrm{e}^{\mathrm{i} h H} R_{1}+B T\right), \quad R_{n}=R\left(F \mathrm{e}^{\mathrm{i} h H} R_{n-1}+B R_{n-2}\right), \\
n=3,4,5, \ldots,
\end{array}
$$

where $H$ is the thickness of the laminate. The output at the nodes in the graph are the amplitudes of the waves in the slab region incident on the interface $x=0$. Thus the total reflection coefficient of the laminate is $\left\langle R_{0}\right\rangle$, where

$$
\left\langle R_{0}\right\rangle=R_{0}+T_{0}\left[B\left(T+R_{2}+R_{4}+\cdots\right)+F \mathrm{e}^{\mathrm{i} h H}\left(R_{1}+R_{3}+R_{5}+\cdots\right)\right] .
$$

Although it is easy to compute the series (4) by using the recurrence relations (3), one can also use the solution of (3) considered as a linear difference equation [6]:

$$
R_{n}=\left[T /\left(\rho_{1}-\rho_{2}\right)\right]\left[\left(R F \mathrm{e}^{\mathrm{i} h H}-\rho_{2}\right) \rho_{1}^{n}+\left(\rho_{1}-R F \mathrm{e}^{\mathrm{i} h H}\right) \rho_{2}^{n}\right],
$$

where

$$
\rho_{1,2}=\frac{1}{2}\left[R F \mathrm{e}^{\mathrm{i} h H} \pm \sqrt{R^{2} F^{2} \mathrm{e}^{2 \mathrm{i} h H}+4 R B}\right]
$$

\section{THE BACKWARD AND FORWARD SCATTERING COEFFICIENTS $B$ AND $F$}

In calculating $B$ and $F$, we consider the matrix material to occupy the whole of space, with the scattering fibers located in the slab region $0 \leqslant x \leqslant H$ (see Figure 1). The scattered displacement field $\mathbf{u}$ in the matrix due to the compressional incident wave $\exp [\mathrm{i}(h x-\omega t)]$ can be split into scalar and vector wave functions in the form

$$
\mathbf{u}=\operatorname{grad} \Phi+\operatorname{curl}\left(\mathbf{e}_{\boldsymbol{z}} \Psi\right),
$$

where $\Phi$ and $\Psi$ satisfy Helmholtz's equation with wavenumbers $h$ and $k$ for compressional and shear waves, respectively:

$$
h=\omega / \alpha, \quad k=\omega / \beta, \quad \alpha=[(\lambda+2 \mu) / \rho]^{1 / 2}, \quad \beta=(\mu / \rho)^{1 / 2} .
$$

If, as in reference [4], we first suppose that there are $N$ fibers, each of radius $a$ centered 
at $O_{i}$ with polar co-ordinates $\left(r_{i}, \theta_{i}\right)(i=1,2, \ldots, N)$, then suppressing the time factor $\exp (-\mathrm{i} \omega t)$ we can write

$$
\Phi=\mathrm{e}^{\mathrm{i} h x}+\sum_{i=1}^{N} \sum_{m=-\infty}^{\infty} A_{i m} \mathrm{H}_{m}\left(h R_{i}\right) \mathrm{e}^{\mathrm{i} m \phi_{i}}, \quad \Psi=\sum_{i=1}^{N} \sum_{m=-\infty}^{\infty} B_{i m} \mathrm{H}_{m}\left(k R_{i}\right) \mathrm{e}^{\mathrm{i} m \phi_{i}},
$$

where $\mathrm{H}_{m}$ is the Hankel function of the first kind and order $m$ and $\left(R_{i}, \phi_{i}\right)$ are the polar co-ordinates of a field point $P$ in the matrix with $O_{i}$ as the origin (see Figure 1).

The transmitted displacement field $\mathbf{u}_{i}^{\prime}$ inside a fiber centered at $O_{i}$ can similarly be written in terms of wave functions $\Phi_{i}^{\prime}$ and $\Psi_{i}^{\prime}$, where

$$
\Phi_{i}^{\prime}=\sum_{m=-\infty}^{\infty} A_{i m}^{\prime} \mathrm{J}_{m}\left(h^{\prime} R_{i}\right) \mathrm{e}^{\mathrm{i} m \phi_{i}}, \quad \Psi_{i}^{\prime}=\sum_{m=-\infty}^{\infty} B_{i m}^{\prime} \mathrm{J}_{m}\left(k^{\prime} R_{i}\right) \mathrm{e}^{\mathrm{i} m \phi_{i}},
$$

with

$$
h^{\prime}=\omega / \alpha^{\prime}, \quad k^{\prime}=\omega / \beta^{\prime}, \quad \alpha^{\prime}=\left[\left(\lambda^{\prime}+2 \mu^{\prime}\right) / \rho^{\prime}\right]^{1 / 2}, \quad \beta^{\prime}=\left(\mu^{\prime} / \rho^{\prime}\right)^{1 / 2} .
$$

$\lambda^{\prime}, \mu^{\prime}$ and $\rho^{\prime}$ are the Lamé constants and density of the material of the fibers, and $\mathrm{J}_{m}$ denotes the Bessel function of order $m$.

In reference [4] four equations are derived from the conditions of continuity of displacement and stress components on the fiber surface of $O_{j}$, which govern the scattering amplitudes $A_{i m}, B_{i m}, A_{i m}^{\prime}$ and $B_{i m}^{\prime}$. As in that paper, the solution of these equations can be written in the form

$$
A_{j n}=(\pi / 4)(a h)^{2}\left(\mathrm{i} P_{n} \Phi_{n}^{j}+Q_{n} \Psi_{n}^{j}\right), \quad B_{j n}=(\pi / 4)(a k)^{2}\left(R_{n} \Phi_{n}^{j}+\mathrm{i} S_{n} \Psi_{n}^{j}\right),
$$

where

$$
\Phi_{n}^{j}=\mathrm{i}^{n} \mathrm{e}^{\mathrm{i} h x_{j}}+\sum_{i=1}^{N}, \sum_{m=-\infty}^{\infty} A_{i, m+n} \mathrm{H}_{m}\left(h r_{i j}\right) \mathrm{e}^{\mathrm{i} m \theta_{i j}}, \quad \Psi_{n}^{j}=\sum_{i=1}^{N} \sum_{m=-\infty}^{\infty} B_{i, m+n} \mathrm{H}_{m}\left(k r_{i j}\right) \mathrm{e}^{\mathrm{i} m \theta_{i j}}
$$

In the above, $\left(r_{i j}, \theta_{i j}\right)$ are polar co-ordinates of $O_{j}$, with respect to $O_{i}$ as origin (see Figure 1) and $\Sigma^{\prime}$ denotes the sum over all the $N$ fibers excepting the $j$ th one. $\Phi_{n}^{j}$ and $\Psi_{n}^{j}$ are evidently related to the field at $O_{j}$ when the $j$ th fiber is assumed absent. The explicit expressions of $P_{n}, Q_{n}, R_{n}$ and $S_{n}$ are very cumbersome to write in general, but in the case of thin fibers (such as glass or carbon fibers in a polymeric matrix) $h a<k a \ll 1$, $h^{\prime} a<k^{\prime} a \ll 1$, the expressions reduce to (cf., [4])

$$
\begin{gathered}
P_{0}=-\frac{\lambda^{\prime}+\mu^{\prime}-\lambda-\mu}{\lambda^{\prime}+\mu^{\prime}+\mu}, \quad S_{0}=0=Q_{0}=R_{0}, \\
P_{1}=-\left(\frac{\mu}{\lambda+2 \mu}\right)^{1 / 2}, \quad Q_{1}=\left(\frac{\lambda+2 \mu}{\mu}\right)^{1 / 2}, \quad R_{1}=S_{1}=\frac{1}{2}\left(\frac{\rho^{\prime}}{\rho}-1\right), \\
P_{2}=R_{2}=-\frac{\mu\left(\mu^{\prime}-\mu\right)}{\mu^{\prime}(\lambda+3 \mu)+\mu(\lambda+\mu)}, \quad-Q_{2}=S_{2}=\frac{(\lambda+2 \mu)\left(\mu^{\prime}-\mu\right)}{\mu^{\prime}(\lambda+3 \mu)+\mu(\lambda+\mu)}, \\
P_{n}=Q_{n}=R_{n}=S_{n}=0, \quad|n| \geqslant 3 .
\end{gathered}
$$

Also, in general, these quantities with negative subscripts are given by

$$
P_{-n}=P_{n}, \quad Q_{-n}=-Q_{n}, \quad R_{-n}=-R_{n}, \quad S_{-n}=S_{n}, \quad n \geqslant 0 .
$$

Under this approximation, equation (12) with the last of equations (14) shows that in equations (9), one needs to consider only five sets of scattering coefficients $A_{i m}$ and $B_{i m}$ 
corresponding to $m=0, \pm 1, \pm 2$. These surviving ten coefficients are given by ten equations, five from each of the sets of equations (12). Finally, as in reference [4], the expressions for $A_{i m}^{\prime}$ and $B_{i m}^{\prime}$, are not required in the subsequent investigation.

We now consider the position $\mathbf{r}_{i} \equiv\left(r_{i}, \theta_{i}\right)$ of $O_{i}$ to be random, with a uniform distribution. As a consequence, the composite behaves as a homogeneous isotropic material (in a cross-section perpendicular to the fibers) on a macroscale. Also, in the case of thin fibers, which is of interest here, when the cross-section of a fiber is almost a point, the position of one with respect to another becomes almost uncorrelated. Hence, as in reference [4], the average total compressional wave function in the regions $x<0$ and $x>H$ becomes, when $N \rightarrow \infty$,

$$
\langle\Phi\rangle=\mathrm{e}^{\mathrm{i} h x}+n_{0} \sum_{m=-\infty}^{\infty} \int_{r_{1} \in S}\left\langle A_{1 m}\right\rangle_{1} \mathrm{H}_{m}\left(h R_{1}\right) \mathrm{e}^{\mathrm{i} m \phi_{1}} \mathrm{~d}_{\mathbf{r}_{1}},
$$

where $n_{0}$ is the number of fibers per unit area and $\left\langle A_{1 m}\right\rangle_{1}$ is the conditional expectation of $A_{1 m}$ when $O_{1}$ is held fixed. The expression for the average total shear wave function $\langle\Psi\rangle$ can be similarly written. In its expression, the term corresponding to the first term on the right side is absent, since there is no incident shear wave.

From equations (12) and (13) one can derive an equation for the conditional expectation $\left\langle A_{1 m}\right\rangle_{1}$ (cf., [4]):

$$
\begin{aligned}
\left\langle A_{1 m}\right\rangle_{1}= & \frac{\pi}{4}(a h)^{2}\left[\mathrm { i } P _ { m } \left\{\mathrm{i}^{m} \mathrm{e}^{\mathrm{i} h x_{1}}+\sum_{n=-\infty}^{\infty} \int_{\left(r_{1}, r_{2}\right) \in S}\left\langle A_{2, m+n}\right\rangle_{12} \mathrm{H}_{n}\left(h r_{12}\right) \mathrm{e}^{\mathrm{i} n \theta_{2 !}} \mathrm{d} \mathbf{r}_{2}\right.\right. \\
& \left.+Q_{m} n_{0} \sum_{n=-\infty}^{\infty} \int_{\left(\mathfrak{r}_{1}, r_{2}\right) \in S}\left\langle B_{2, m+n}\right\rangle_{12} \mathrm{H}_{n}\left(k r_{12}\right) \mathrm{e}^{\mathrm{i} n \theta_{21}} \mathrm{~d} \mathbf{r}_{2}\right] .
\end{aligned}
$$

In equations (16) and (17), $S$ denotes the slab region within which the fibers lie and $\left\langle A_{2 m}\right\rangle_{12}$ and $\left\langle B_{2 m}\right\rangle_{12}$ denote the conditional expectation of $A_{2 m}$ and $B_{2 m}$ with fibers $O_{1}$ and $O_{2}$ held fixed. An expression for $\left\langle B_{1 m}\right\rangle_{1}$ can similarly be derived and its form is similar to that of equation (17) except that $h$ outside the square brackets is to be replaced by $k$ and i $P_{n}, Q_{n}$ by $R_{n}, \mathrm{i} S_{n}$, respectively. In the hierarchical equation (17), we break the hierarchy by invoking the "quasi-crystalline" approximation

$$
\left\langle A_{\text {im }}\right\rangle_{i j}=\left\langle A_{\text {im }}\right\rangle_{i},\left\langle B_{\text {im }}\right\rangle_{i j}=\left\langle B_{\text {im }}\right\rangle_{i}, \quad i \neq j .
$$

Briefly, this means that had the fibers formed a lattice, as in a crystal, the average obtained by holding two fibers fixed would be the same as that by holding one fiber fixed. In a dense system, such as the one under discussion, where there is closeness to a lattice structure, equation (18) is expected to be approximately true. With this approximation, equation (17) and the analogous one for $\left\langle B_{i m}\right\rangle_{1}$ form a coupled integro-difference equation. We now seek coherent waves propagating in the composite slab region and assume that the solution is of the form

$$
\left\langle A_{i m}\right\rangle_{i}=\mathrm{i}^{m}\left(X_{m} \mathrm{e}^{\mathrm{i} K_{x_{i}}}+\bar{X}_{m} \mathrm{e}^{-\mathrm{i} X_{x_{i}}}\right), \quad\left\langle B_{i m}\right\rangle_{i}=\mathrm{i}^{m+1}\left(Y_{m} \mathrm{e}^{\mathrm{i} K x_{i}}+\bar{Y}_{m} \mathrm{e}^{-\mathrm{i} \bar{K} x_{i}}\right) .
$$

Inserting these expressions in equation (17), we come across surface integrals which can be converted by Green's identity:

$$
\begin{aligned}
\int_{S} \mathrm{e}^{\mathrm{i} K x_{2}} \mathrm{H}_{n}\left(h r_{12}\right) \mathrm{e}^{\mathrm{i} n \theta_{21}} \mathrm{~d} \mathbf{r}_{2}= & \frac{(-1)^{n} \mathrm{e}^{\mathrm{i} K x_{1}}}{K^{2}-h^{2}} \int_{\partial s}\left[\mathrm{e}^{\mathrm{i} \kappa\left(x_{2}-x_{1}\right)} \frac{\partial}{\partial n}\left\{\mathrm{H}_{n}\left(h r_{\mathrm{i} 2}\right) \mathrm{e}^{\mathrm{i} n \theta_{12}}\right\}\right. \\
& \left.-\mathrm{H}_{n}\left(h r_{12}\right) \mathrm{e}^{\mathrm{i} n \theta_{12}} \frac{\partial}{\partial n}\left\{\mathrm{e}^{\mathrm{i} K\left(x_{2}-x_{1}\right)}\right\}\right] \mathrm{d} \mathbf{r}_{2} .
\end{aligned}
$$


We take care to note that the boundary $\partial S$ is such that the fiber at $O_{2}$ does not go outside of $S$; nor does it penetrate the fiber at $O_{1}$. This is shown in Figure 3 by the dashed lines.

The contribution from the integral along the dashed circle in expression (20) is easily found to be (as in reference [4])

$$
2 \pi a(-\mathrm{i})^{-n}\left[\mathrm{~J}_{n}(2 K a) \frac{\partial}{\partial a} \mathbf{H}_{n}(2 h a)-\mathbf{H}_{n}(2 h a) \frac{\partial}{\partial a} \mathrm{~J}_{n}(2 K a)\right] .
$$

For the contributions coming from the straight portions $x=a, H-a$ we use the result

$$
\mathbf{H}_{n}(h r) \mathrm{e}^{\mathrm{i} n \theta}=\frac{(-\mathrm{i})^{n}}{\mathrm{i} \pi} \int_{-\infty}^{\infty} \mathrm{e}^{-p|y|} \mathrm{e}^{\mathrm{i} \xi x}\left(\frac{h}{\xi+p}\right)^{n} \frac{\mathrm{d} \xi}{p},
$$

where $p(\xi)=\left(\xi^{2}-h^{2}\right)^{1 / 2}$ if $\xi>h$ or else $-\mathrm{i}\left(h^{2}-\xi^{2}\right)^{1 / 2}$. This formula effectively expresses cylindrical waves in terms of plane waves and can be proved along the lines described in reference [7]. Typically, the integrals along the straight portions depend on the integral

$$
\int_{y_{2}-y_{1}=-\infty}^{\infty} \mathbf{H}_{n}\left(h r_{12}\right) \mathrm{e}^{\mathrm{in} \theta \theta_{12}} \mathrm{~d}\left(y_{2}-y_{1}\right)
$$

which, on using equation (22), equals

$$
\frac{2(-\mathrm{i})^{n}}{\mathrm{i} \pi} \int_{-\infty}^{\infty} \mathrm{e}^{\mathrm{i} \xi\left(x_{2}-x_{1}\right)}\left(\frac{h}{\xi+p}\right)^{n} \frac{\mathrm{d} \xi}{\xi^{2}-h^{2}} .
$$

The contour of integration in equation (22) is correctly chosen from the considerations for the Hankel function of the second kind given in reference [8]. Upon assuming a vanishingly small imaginary part of $\omega$, the contour along the $\xi$-axis takes up the position relative to the branch cuts as shown in Figure 4(a). Distorting the contour to the forms 4(b) or 4(c), we obtain, for equation (24),

$$
\frac{2 \mathrm{i}^{\mathrm{n}}}{\mathrm{i} \pi}\left[\frac{\mathrm{i} \pi}{h} \mathrm{e}^{\mathrm{i} h\left(x_{1}-x_{2}\right)}+I h_{n}\right]
$$

for $x_{2}<x_{1}$, where

$$
\begin{aligned}
I h_{n}= & \int_{0}^{h} \mathrm{e}^{\mathrm{i} \xi\left|x_{\mathrm{x}}-x_{2}\right|}\left[\left(\frac{h}{\xi-\mathrm{i} \sqrt{h^{2}-\xi^{2}}}\right)^{n}-\left(\frac{h}{\xi+\mathrm{i} \sqrt{h^{2}-\xi^{2}}}\right)^{n}\right] \frac{\mathrm{d} \xi}{h^{2}-\xi^{2}} \\
& +\mathrm{i}^{-n+1} \int_{0}^{\infty} \mathrm{e}^{-\eta\left|x_{1}-x_{2}\right|}\left[\left(\frac{h}{\eta+\sqrt{\eta^{2}-h^{2}}}\right)^{n}-\left(\frac{h}{\eta-\sqrt{\eta^{2}+h^{2}}}\right)^{n}\right] \frac{\mathrm{d} \eta}{\eta^{2}+h^{2}},
\end{aligned}
$$

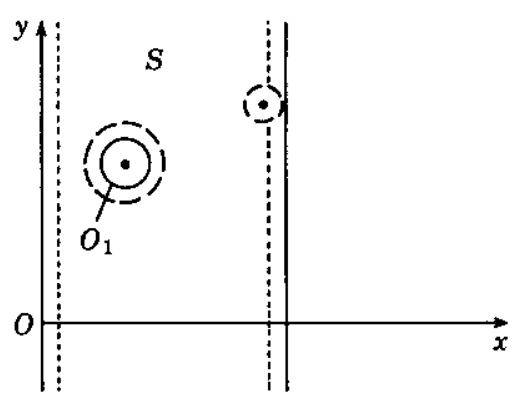

Figure 3. The boundary (dashed lines) $\partial S$ of the region $S$. 


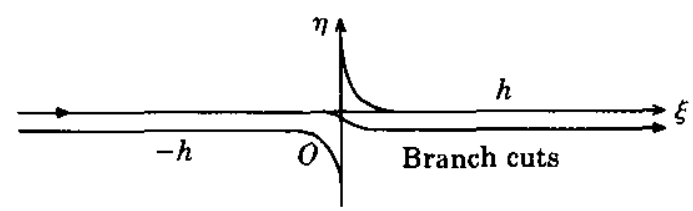

(a)

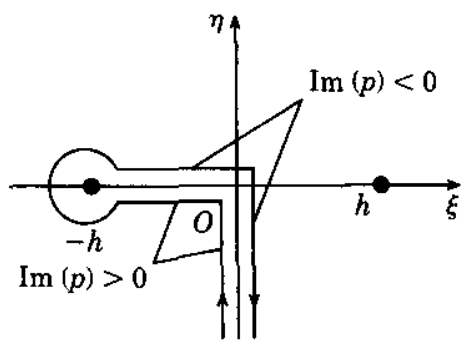

(b)

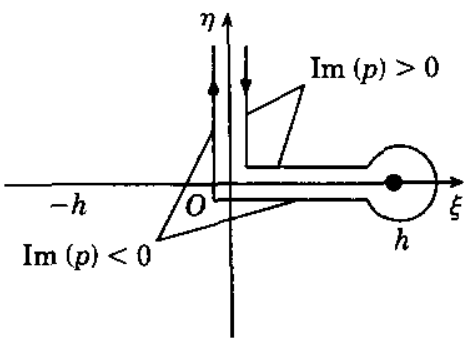

(c)

Figure 4. (a) Original contour of integration in equations (22); (b) distorted contour when $x_{2}<x_{1}$; (c) distorted contour when $x_{2}>x_{1}$.

and, for $x_{2}>x_{1}$,

$$
\frac{2(-\mathrm{i})^{n}}{\mathrm{i} \pi}\left[\frac{\mathrm{i} \pi}{h} \mathrm{e}^{\mathrm{i} h\left(x_{2}-x_{1}\right)}-I h_{n}\right] .
$$

An important property of the integral $I h_{n}$ is that it is odd in $n$ :

$$
\mathrm{i} h_{-n}=-I h_{n} \text {. }
$$

Using the results (20), (21), (25), (27) and the properties (15) and (27), we obtain, from equations (17) and (19) after some manipulations, that

$$
\begin{aligned}
\left(x_{m}\right. & \left.+x_{-m}\right) \mathrm{e}^{\mathrm{i} K x_{1}}+\left(\bar{x}_{m}+\bar{x}_{-m}\right) \mathrm{e}^{-\mathrm{i} R x_{1}}=-\left[c P_{m} \sum_{n=0}^{\infty} \epsilon_{n}\left(F_{n-m}+F_{n+m}\right)\left(x_{n}+x_{-n}\right)\right. \\
& \left.-c Q_{m} \sum_{n=0}^{\infty}\left(G_{n-m}-G_{n+m}\right)\left(y_{n}-y_{-n}\right)\right] \mathrm{e}^{\mathrm{i} K x_{1}} \\
& -\left[c P_{m} \sum_{n=0}^{\infty}(-1)^{n-m} \epsilon_{n}\left(\bar{F}_{n-m}+\bar{F}_{n+m}\right)\left(\bar{x}_{n}+\bar{x}_{-n}\right)\right. \\
& \left.-c Q_{m} \sum_{n=0}^{\infty}(-1)^{n-m}\left(\bar{G}_{n-m}-\bar{G}_{n+m}\right)\left(\bar{y}_{n}-\bar{y}_{-n}\right)\right] \mathrm{e}^{-\mathrm{i} R x_{1}} \\
& +\frac{\mathrm{i} \pi}{2}(a h)^{2} P_{m}\left[1+2 \mathrm{i} n_{0}\left\{\frac{\mathrm{e}^{\mathrm{i} K a}}{h(K-h)} \sum_{n=0}^{\infty} \epsilon_{n}\left(x_{n}+x_{-n}\right)-\frac{\mathrm{e}^{-\mathrm{i} K a}}{h(\bar{K}+h)} \sum_{n=0}^{\infty} \epsilon_{n}\left(\bar{x}_{n}+\bar{x}_{-n}\right)\right\} \mathrm{e}^{-\mathrm{i} h a}\right] \mathrm{e}^{\mathrm{i} h x_{1}} \\
& +\pi(a h)^{2} P_{m} n_{0}\left\{\frac{\mathrm{e}^{\mathrm{i} K(H-a)}}{h(K+h)} \sum_{n=0}^{\infty}(-1)^{n-m} \epsilon_{n}\left(x_{n}+x_{-n}\right)\right. \\
& \left.-\frac{\mathrm{e}^{-\mathrm{i} R(H-a)}}{h(\bar{K}-h)} \sum_{n=0}^{\infty}(-1)^{n-m} \epsilon_{n}\left(\bar{x}_{n}+\bar{x}_{-n}\right)\right\} \mathrm{e}^{\mathrm{i} h\left(H-a-x_{1}\right)},
\end{aligned}
$$


A. BAGCHI AND S. K. BOSE

where $\epsilon_{0}=1 / 2$ and $\epsilon_{n}=1$ for $n \geqslant 1$. In equation (29) we have dropped the terms containing integrals of the type $I h_{n}$. This is justified because these terms are multiplied by combinations $x_{n}-x_{-n}, \bar{x}_{n}-\bar{x}_{-n}, y_{n}+y_{-n}$ or $\bar{y}_{n}+\bar{y}_{-n}$, and these terms vanish for the coherent compressional waves that we are seeking.

If we treat the equation for $\left\langle B_{\mathrm{Im}}\right\rangle_{\mathrm{I}}$ analogous to equation (17), we obtain

$$
\begin{aligned}
& \left(y_{m}-y_{-m}\right) \mathrm{e}^{\mathrm{i} K x_{1}}+\left(\bar{y}_{m}-\bar{y}_{-m}\right) \mathrm{e}^{-\mathrm{i} R x_{1}}=\left[c R_{m} \sum_{n=0}^{\infty} \epsilon_{n}\left(F_{n-m}+F_{n+m}\right)\left(x_{n}+x_{-n}\right)\right. \\
& \left.-c S_{m} \sum_{n=0}^{\infty}\left(G_{n-m}-G_{n+m}\right)\left(y_{n}-y_{-n}\right)\right] \mathrm{e}^{\mathrm{i} K x_{1}} \\
& +\left[c R_{m} \sum_{n=0}^{\infty}(-1)^{n-m} \epsilon_{n}\left(\bar{F}_{n-m}+\bar{F}_{n+m}\right)\left(x_{n}+x_{-n}\right)\right. \\
& \left.\quad-c S_{m} \sum_{n=0}^{\infty}(-1)^{n-m}\left(\bar{G}_{n-m}-\bar{G}_{n+m}\right)\left(y_{n}-y_{-n}\right)\right] \mathrm{e}^{-\mathrm{i} R x_{1}} \\
& \quad-\frac{\mathrm{i} \pi}{2}(a k)^{2} R_{m}\left[1+2 \mathrm{i} n_{0}\left\{\frac{\mathrm{e}^{\mathrm{i} K a}}{h(K-h)} \sum_{n=0}^{\infty} \epsilon_{n}\left(x_{n}+x_{-n}\right)-\frac{\mathrm{e}^{-\mathrm{i} K a}}{h(\bar{K}+h)} \sum_{n=0}^{\infty} \epsilon_{n}\left(\bar{x}_{n}+\bar{x}_{-n}\right)\right\} \mathrm{e}^{-\mathrm{i} h a}\right] \mathrm{e}^{\mathrm{i} h x_{1}} \\
& -\pi(a k)^{2} R_{m} n_{0}\left\{\frac{\mathrm{e}^{\mathrm{j} K(H-a)}}{h(K+h)} \sum_{n=0}^{\infty}(-1)^{n-m} \epsilon_{n}\left(x_{n}+x_{-n}\right)\right. \\
& \left.-\frac{\mathrm{e}^{-\mathrm{i} K(H-a)}}{h(\bar{K}-h)} \sum_{n=0}^{\infty}(-1)^{n-m} \epsilon_{n}\left(\bar{x}_{n}+\bar{x}_{-n}\right)\right\} \mathrm{e}^{\mathrm{i} h\left(H-a-x_{1}\right)} .
\end{aligned}
$$

The quantities $F_{n}$ and $Q_{n}$ arise from equation (21) as in reference [4], and are given by

$$
F_{n}=\frac{\mathrm{i} \pi}{2} \frac{a h^{2}}{K^{2}-h^{2}}\left\{\mathrm{~J}_{n}(2 K a) \frac{\partial}{\partial a} \mathrm{H}_{n}(2 h a)-\mathrm{H}_{n}(2 h a) \frac{\partial}{\partial a} \mathrm{~J}_{n}(2 K a)\right\}=\frac{1}{1-K^{2} / h^{2}}\left(\frac{K}{h}\right)^{|n|},
$$

for thin fibers. $G_{n}$ has a similar expression with $h$ replaced by $k$ in equation (31). In $\bar{F}_{n}$ and $\bar{G}_{n}, K$ and $G$ are to be replaced by $\bar{K}$ and $\bar{G}$. All these quantities are even in $n$. Equating the amplitudes of the different waves in equations (29) and (30), we obtain

$$
\begin{gathered}
2 \mathrm{in} n_{0} \mathrm{e}^{-\mathrm{i} h a}\left[\frac{\mathrm{e}^{\mathrm{i} K a}}{h(K-h)} \sum_{n=0}^{\infty} \epsilon_{n}\left(x_{n}+x_{-n}\right)-\frac{\mathrm{e}^{-\mathrm{i} R a}}{h(\bar{K}+h)} \sum_{n=0}^{\infty} \epsilon_{n}\left(\bar{x}_{n}+\bar{x}_{-n}\right)\right]=-1, \\
\frac{\mathrm{e}^{\mathrm{i} K(H-a)}}{h(K+h)} \sum_{n=0}^{\infty}(-1)^{n} \epsilon_{n}\left(x_{n}+x_{-n}\right)-\frac{\mathrm{e}^{-\mathrm{i} R(H-a)}}{h(\bar{K}-h)} \sum_{n=0}^{\infty}(-1)^{n} \epsilon_{n}\left(\bar{x}_{n}+\bar{x}_{-n}\right)=0 \\
x_{m}+x_{-m}=-c P_{m} \sum_{n=0}^{\infty} \epsilon_{n}\left(F_{n-m}+F_{n+m}\right)\left(x_{n}+x_{-n}\right)-c Q_{m} \sum_{n=0}^{\infty}\left(G_{n-m}-G_{n+m}\right)\left(y_{n}-y_{-n}\right), \\
y_{m}-y_{-m}=c R_{m} \sum_{n=0}^{\infty} \epsilon_{n}\left(F_{n-m}+F_{n+m}\right)\left(x_{n}+x_{-n}\right)-c S_{m} \sum_{n=0}^{\infty}\left(G_{n-m}-G_{n+m}\right)\left(y_{n}-y_{-n}\right),
\end{gathered}
$$


and upon setting

$$
(-1)^{n} \bar{x}_{n}=\bar{x}_{n}, \quad(-1)^{n} \bar{y}_{n}=\bar{y}_{n},
$$

the quantities $\bar{x}_{n}+\bar{x}_{-n}$ and $\bar{y}_{n}-\bar{y}_{-n}$ are seen to satisfy the same set of equations as equations (33).

For thin fibers, the system of equations (33) effectively yields a fifth order determinantal equation for the wavenumber $K$, which gives (cf., [4])

$$
\frac{K^{2}}{h^{2}}\left[1-c P_{2}\left(1-\frac{k^{2}}{h^{2}}\right)-2 c^{2} P_{0} P_{2}\right]=\left(1+2 c P_{1}\right)\left(1+c P_{0}\right)\left[1+c P_{2}\left(1+\frac{k^{2}}{h^{2}}\right)\right]
$$

Hence if $K$ is the positive square root of equation (35)

$$
\bar{K}=K \text {. }
$$

When equation (35) is satisfied only four of equations (33) are independent and one can solve them for $x_{1}+x_{-1}$ and $x_{2}+x_{-2}$, in terms of $x_{0}$, to obtain

$$
x_{1}+x_{-1}=\chi_{1} x_{0}, \quad x_{2}+x_{-2}=x_{2} x_{0}
$$

where

$$
\begin{gathered}
\chi_{1}=\frac{\left\{1+\left(K^{2} / h^{2}\right)+c P_{0}\right\}\left\{P_{1}+c S_{1}\left(P_{1}+Q_{1}\right)\right\}-(h / k) Q_{1}\left\{1-\left(K^{2} / h^{2}\right)+c P_{0}\right\}}{P_{0}(K / h)\left(1+c S_{1}+c Q_{1} h / k\right)} \\
\chi_{2}=\frac{\left\{1+\left(K^{2} / h^{2}\right)+c P_{0}\right\}\left(c+c S_{1}\right)+c\left\{1-\left(K^{2} / h^{2}\right)+c P_{0}\right\}\left\{P_{1}+c S_{1}\left(P_{1}+Q_{1}\right)\right\}}{c P_{0}\left(K^{2} / h^{2}\right)\left(1+c S_{1}+c Q_{1} h / k\right)} .
\end{gathered}
$$

Similarly, we have

$$
\bar{x}_{1}+\bar{x}_{-1}=\chi_{1} \bar{x}_{0}, \quad \bar{x}_{2}+\bar{x}_{-2}=\chi_{2} \bar{x}_{0} .
$$

Finally, equation (29) yields

$$
\bar{x}_{0}=\frac{K-h}{K+h} \frac{1-\chi_{1}+\chi_{2}}{1+\chi_{1}+\chi_{2}} \mathrm{e}^{2 \mathrm{i} K(H-a)} x_{0}
$$

and

$$
2 \mathrm{i} n_{0} \mathrm{e}^{-\mathrm{i} h a} x_{0}\left[\frac{\mathrm{e}^{\mathrm{i} K a}}{h(K-h)}\left(1+\chi_{1}+\chi_{2}\right)-\mathrm{e}^{\mathrm{i} K(2 H-3 a)} \frac{K-h}{h(K+h)^{2}} \frac{\left(1-\chi_{1}+\chi_{2}\right)^{2}}{\left(1+\chi_{1}+\chi_{2}\right)}\right]=1 .
$$

Equations (37)-(41) yield the amplitudes of the forward and backward scattered coherent compressional waves.

\section{THE BACKSCATTERED AND THE FORWARD-SCATTERED WAVES}

The average wave function $\langle\Phi\rangle$ given by equations (12), (15) and (32) can be written as

$$
\langle\Phi\rangle=\mathrm{e}^{\mathrm{i} h x}+n_{0} \sum_{m=-\infty}^{\infty}(-\mathrm{i})^{m} \int_{\mathrm{r}_{l} \in S}\left(x_{m} \mathrm{e}^{\mathrm{i} K x_{1}}+\bar{x}_{m} \mathrm{e}^{-\mathrm{i} R x_{1}}\right) \mathrm{H}_{m}\left(h R_{1}\right) \mathrm{e}^{\mathrm{i} m \psi_{1}} \mathrm{dr}_{1},
$$

where $\psi_{1}=-\pi+\phi_{1}$ is the polar angle of $O_{1}$ with respect to $P$ as origin. As in the case of treating equation (17) by Green's identity (20), we can evaluate the above integral and, after some simplification, obtain the form

$$
\langle\Phi\rangle=\mathrm{e}^{\mathrm{i} h x}+B \mathrm{e}^{-\mathrm{i} h x},
$$


for the region $x<0$, where the back-scattering coefficient $B$ is given by

$$
B=\left[2 \mathrm{in} n_{0} / h(K+h)\right] \mathrm{e}^{\mathrm{i} h a}\left[\mathrm{e}^{\mathrm{i} K a}-\mathrm{e}^{\mathrm{i} K(2 H-3 a)}\right]\left(1-\chi_{1}+\chi_{2}\right) x_{0} .
$$

Similarly for the region $x>H$, we obtain

$$
\langle\Phi\rangle=F \mathrm{e}^{\mathrm{i} h x},
$$

where the forward-scattering (transmission) coefficient is given by

$$
\begin{aligned}
F=1 & +\frac{2 \mathrm{i} n_{0}}{h}\left[\frac{1}{K-h}\left\{\mathrm{e}^{\mathrm{i}(K-h) a}-\mathrm{e}^{\mathrm{i}(K-h)(H-a)}\right\}\left(1+\chi_{1}+\chi_{2}\right)\right. \\
& \left.-\frac{K-h}{(K+h)^{2}} \mathrm{e}^{2 \mathrm{i} K(H-a)}\left\{\mathrm{e}^{-\mathrm{i}(K+h) a}-\mathrm{e}^{\mathrm{i}(K+h)(H-a)}\right\} \frac{\left(1-\chi_{1}+\chi_{2}\right)^{2}}{1+\chi_{1}+\chi_{2}}\right] x_{0} .
\end{aligned}
$$

Under the thin fiber approximation, we can assume that $K a$ is also much smaller than 1, and hence the expressions for $B, F$ and $x_{0}$ undergo further obvious simplification (exponentials of iha and $\mathrm{i} K a \approx 1$ ).

\section{A NUMERICAL EXAMPLE}

We consider a carbon-epoxy composite with the following properties: $\rho=1.20 \mathrm{~g} / \mathrm{cm}^{3}$, $E=3.50 \times 10^{4} \mathrm{~kg} / \mathrm{cm}^{2}$ and $\mu=1.24 \times 10^{4} \mathrm{~kg} / \mathrm{cm}^{2}$, so that $\lambda=2.57 \times 10^{4} \mathrm{~kg} / \mathrm{cm}^{2}$. For the properties of carbon fibers in the transversal direction, we take $\rho^{\prime}=1.78 \mathrm{~g} / \mathrm{cm}^{3}$, $E^{\prime}=20.60 \times 10^{4} \mathrm{~kg} / \mathrm{cm}^{2}$ and $\mu^{\prime}=6.70 \times 10^{4} \mathrm{~kg} / \mathrm{cm}^{2}$, so that $\lambda^{\prime}=14.13 \times 10^{4} \mathrm{~kg} / \mathrm{cm}^{2}$. Finally, for water, we have $\rho_{0}=1.0 \mathrm{~g} / \mathrm{cm}^{3}$ and $\alpha_{0}=1.524 \mathrm{~cm} / \mu \mathrm{s}$. The value of $\alpha$ as calculated from $\lambda, \mu$ and $\rho$ is $2.03 \mathrm{~cm} / \mu \mathrm{s}$.

A composite laminate consists of several plies. If we suppose that each ply is $10^{-2} \mathrm{~cm}$ thick, a two-ply laminate has $H=2 \times 10^{-2} \mathrm{~cm}$. The results of computation of the magnitude (in $\mathrm{dB}$ ) and phase in the range $-\pi, \pi$ of $B, F$ and $\left\langle R_{0}\right\rangle$ for $0.1,0.5$ and $1.0 \mathrm{MHz}$ frequencies are presented in Table 1 . Higher frequencies are not considered, because a recent investigation by Bose [9] indicates that the propagation phenomenon may then be

\begin{tabular}{|c|c|c|c|c|c|c|c|}
\hline \multirow{2}{*}{$\frac{c}{0.60}$} & \multirow{2}{*}{$\begin{array}{c}\begin{array}{c}\text { Scattering } \\
\text { coefficient }\end{array} \\
\bar{R}_{0} \\
B \\
F \\
\left\langle R_{0}\right\rangle\end{array}$} & \multicolumn{2}{|c|}{$0.1 \mathrm{MHz}$} & \multicolumn{2}{|c|}{$0.5 \mathrm{MHz}$} & \multicolumn{2}{|c|}{$1.0 \mathrm{MHz}$} \\
\hline & & $\begin{array}{r}-6.2959 \\
-19.2117 \\
-0.0523 \\
-11.9239\end{array}$ & $\begin{array}{l}-1.0042 \\
-1.2821 \\
-0.3304 \\
-1.2025\end{array}$ & $\begin{array}{l}-0.9374, \\
-8.4986, \\
-0.6612 \\
-3.6871\end{array}$ & $\begin{array}{l}-0 \cdot 1020 \\
-0 \cdot 2158 \\
-1 \cdot 7402 \\
-0 \cdot 1671\end{array}$ & $\begin{array}{r}-3 \cdot 2786, \\
-14.6895 \\
-0 \cdot 1498 \\
-7 \cdot 8060\end{array}$ & $\begin{array}{l}0.7078 \\
1.0715 \\
2.7351 \\
0.9542\end{array}$ \\
\hline 0.65 & $\begin{array}{c}\bar{R}_{0} \\
B \\
F \\
\left\langle R_{0}\right\rangle\end{array}$ & $\begin{array}{r}-6.3589 \\
-18.5256 \\
-0.0619 \\
-11.9102\end{array}$ & $\begin{array}{l}-1.0191 \\
-1.3033 \\
-0.3516 \\
-1.2212\end{array}$ & $\begin{array}{l}-0.8507, \\
-7.4568, \\
-0.8603, \\
-2.8908,\end{array}$ & $\begin{array}{l}-0.1531 \\
-0.3336 \\
-1.8580 \\
-0.2548\end{array}$ & $\begin{array}{l}-1 \cdot 5041 \\
-9 \cdot 8834 \\
-0.4702 \\
-4.4849\end{array}$ & $\begin{array}{l}0.4111 \\
0.7749 \\
2.4385 \\
0.6344\end{array}$ \\
\hline 0.70 & $\begin{array}{c}\bar{R}_{0} \\
B \\
F \\
\left\langle R_{0}\right\rangle\end{array}$ & $\begin{array}{r}-6.4315 \\
-17.9445 \\
-0.0706 \\
-11.9136\end{array}$ & $\begin{array}{l}-1.0335 \\
-1.3233 \\
-0.3716 \\
-1.2390\end{array}$ & $\begin{array}{l}-0.7858, \\
-6.6081 \\
-1.0700 \\
-2.6695\end{array}$ & $\begin{array}{l}-0.1985 \\
-0.4429 \\
-1.9673 \\
-0.3347\end{array}$ & $\begin{array}{l}-0.8316 \\
-6.8207 \\
-1.0122 \\
-2.7944\end{array}$ & $\begin{array}{l}0.2231 \\
0.4912 \\
2 \cdot 1548 \\
0.3737\end{array}$ \\
\hline
\end{tabular}
of an entirely different nature.

TABLE 1

Magnitude (in $d B$ ) and phase of $\bar{R}_{0}, B, F$ and $\left\langle R_{0}\right\rangle$ for different frequencies 
The tabulated data show no particular trend with respect to change in frequency and this originates from the ultra high frequency exp (iKh) term in the expressions for $B, F$ and $x_{0}$. This effect will be accentuated in thicker laminates. However, for a given frequency the amplitudes of $B,\left\langle R_{0}\right\rangle$ and $F$ are in increasing order of magnitude.

In this table we have also presented the result of computing the reflection coefficient $\bar{R}_{0}$, when we model the laminate by a homogeneous lamina with mean density $\langle\rho\rangle=c \rho^{\prime}+(1-c) \rho$ and a propagation property given by equation (31). The expression for $\bar{R}_{0}$ is easily found to be

$$
\vec{R}_{0}=\frac{(\langle\rho\rangle \alpha h)^{2}-\left(\rho_{0} \alpha_{0} K\right)^{2}}{\left(\rho_{0} \alpha_{0} K\right)^{2}+(\langle\rho\rangle \alpha h)^{2}+2 \mathrm{i} \rho_{0}\langle\rho\rangle \alpha_{0} \alpha h K \cot (K H)} .
$$

The amplitude of $\bar{R}_{0}$ clearly turns out to be much larger than that of $\left\langle R_{0}\right\rangle$.

\section{THE BACKSCATTERED REFLECTION COEFFICIENT AND ITS ESTIMATION}

An ultrasonic backscattering experiment carries with it the signature of the local properties of the specimen. As with a slight change in $H$ (on a scale much larger than the wavelengths), a slight change in the distribution of the fibers may alter the backscattered reflection significantly. Thus, when different locations of a specimen are probed, the backscattered signals appear as random [3]. In other words, the backscattered reflection coefficient must be spatially random, with a mean $\left\langle R_{0}\right\rangle$, which we have theoretically investigated in sections 2-4.

Since probing each and every point of a given laminate is not an attractive procedure, we now explore the possibility of estimating the reflection coefficient $R_{0}(\mathbf{r})$ at a point $\mathbf{r}$ of the laminate, when its values at points $\mathbf{r}_{i}(i=1,2, \ldots, M)$ are experimentally known. A problem of this nature occurs in mining geostatistics, where the statistical estimation procedure often used is called kriging [5].

Making a change of notation, we write $Z(\omega)$ for the magnitude (in $\mathrm{dB}$ ) of the backscattered reflection coefficient at $\mathbf{r}(\omega$ is the circular frequency of the ultrasound, which is centered in a range $\left.\left(\omega_{1}, \omega_{2}\right)\right)$. Similarly, we write $Z_{i}(\omega)$ for those measured at $\mathbf{r}_{i}$. In the theory of kriging, $Z(\omega)$ is estimated by $Z^{*}(\omega)$, given by the linear relationship

$$
Z^{*}(\omega)=\sum_{j=1}^{M} \alpha_{j}(\omega) Z_{j}(\omega)
$$

Next it is assumed that $Z$ is spatially stationary, having mean $m(\omega)=20 \log _{10}\left|\left\langle R_{0}\right\rangle\right|$, so that taking the expectation of equations (47) gives

$$
\sum_{j=1}^{M} \alpha_{j}(\omega)=1
$$

This makes the estimate unbiased. The $\alpha_{j}(\omega)$ are obtained by making the estimation error variance

$$
\mathrm{E}\left[\int_{\omega_{1}}^{\omega_{2}}\left\{Z^{*}(\omega)-\sum_{j=1}^{M} \alpha_{j}(\omega) Z_{j}(\omega)\right\}^{2} \mathrm{~d} \omega\right]
$$

a minimum. This can be written as

$$
\int_{\omega_{1}}^{\omega_{2}}\left[C\left(Z^{*}, Z^{*}\right)-2 \sum_{j=1}^{M} \alpha_{j}(\omega) C\left(Z^{*}, Z_{j}\right)+\sum_{i=1}^{M} \sum_{j=1}^{M} \alpha_{i}(\omega) \alpha_{j}(\omega) C\left(Z_{i}, Z_{j}\right)\right] \mathrm{d} \omega
$$


where the covariances are defined by

$$
\begin{gathered}
C\left(Z^{*}, Z^{*}\right)=\mathrm{E}\left[\left(Z^{*}-m\right)^{2}\right], \quad C\left(Z^{*}, Z_{j}\right)=\mathrm{E}\left[\left(Z^{*}-m\right)\left(Z_{j}-m\right)\right], \\
C\left(Z_{i}, Z_{j}\right)=\mathrm{E}\left[\left(Z_{i}-m\right)\left(Z_{j}-m\right)\right] .
\end{gathered}
$$

In order to simplify the analysis we assume that the coefficients $\alpha_{j}$ are independent of $\omega$. Using the Lagrange method of undetermined multipliers on equation (49), subject to condition (48), we obtain the system of equations

$$
\sum_{j=1}^{M} \alpha_{j} \bar{C}\left(Z_{i}, Z_{j}\right)-l=\bar{C}\left(Z^{*}, Z_{i}\right), \quad i=1,2, \ldots, M, \quad \sum_{n=0}^{M} \alpha_{j}=1,
$$

to determine the $\alpha_{j}$ and the undetermined multiplier $l$. In expressions (51), the bar above $C$ denotes integration of the corresponding covariance function with respect to $\omega$. From the first set of equations (51) and (49), it follows that the "kriging variance" is

$$
\sigma_{K}^{2}=\bar{C}\left(Z^{*}, Z^{*}\right)+l-\sum_{j=1}^{M} \alpha_{j} \bar{C}\left(Z^{*}, Z_{j}\right)
$$

$\sigma_{K}$ gives a measure of the dispersion of $Z$ from the linear combination (47). An interesting consequence of equation (52) is that if $r$ coincides with any of the data points $\mathbf{r}_{i}, \sigma_{K}$ becomes zero by virtue of the first set of equations (51). Hence, in a probabilistic sense, "kriging" (equations (47)) is an interpolation over the given data points.

In view of the assumption of stationarity of the stochastic process, it is envisaged in equation (50) that the said covariance function depends only on the vector joining the two points. If, in addition, the distribution is isotropic, the covariance function will be a function of the distance between the two points. A number of standard computer programs are available for kriging, and are used extensively in geographical information systems (GIS).

\section{CONCLUSIONS}

In performing backscattering experiments with ultrasonic waves to evaluate the quality of composite laminates, backscattered amplitude shows a random behavior with change of position in a $c$-scan. In this paper we have evaluated the average reflection coefficient in terms of the fiber concentration and the properties of the fiber and matrix phases. Finally, we have discussed a method for estimating the actual random backscattered reflection coefficient at any location from experimental data at a given grid of locations of the laminate.

\section{REFERENCES}

1. Y. BAR-Cohen and L. CRANe 1982 Materials Evaluation 40, 970-975. Acoustic-backscattering imaging of subcritical flaws in composites.

2. Y. BAR-COHEN and A. K. MAL 1989 Twelfth World Conference on Nondestructive Testing, Amsterdam, Holland. NDE of composite of laminates using leaky Lamb wave pulses.

3. Y. BAR-COHEN, Personal communication.

4. S. K. Bose and A. K. MAL 1974 Journal of the Mechanics and Physics of Solids 22, 217-229. Elastic waves in fiber-reinforced composite.

5. A. G. Journel and CH. J. HuiJbregts 1978 Mining Geostatistics. New York: Academic Press.

6. L. M. MiLne-Thomson 1960 The Calculus of Finite Differences. London: Macmillan.

7. V. R. ThiruvenKatachar and K. Viswanathan 1965 Journal of Mathematical Mechanics 14, 541-571. Dynamic response of an elastic half space with cylindrical cavity to time-dependent surface tractions over the boundary of the cavity. 
8. M. Ewing, W. S. JARdetzky and F. Press 1957 Elastic Waves in Layered Media. London: McGraw-Hill.

9. S. K. Bose 1990 Research Report, National Centre for Basic Sciences, Calcutta. Ultra high frequency longitudinal shear waves in a uni-directional fiber reinforced composite. 